\title{
Computational Biology
}

\section{Holger Husi}

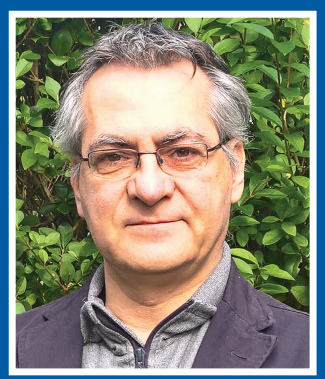

Holger Husi is a senior research fellow in Bioinformatics and Biostatistics at the University of the Highlands and Islands and the University of Glasgow. Originally from Switzerland, where he completed his doctoral training at Novartis, Basel, and the Swiss Federal Institute of Technology (ETH), Zurich, in immunology and the mode of action of immunosuppressants, he specialized in large-scale biosystems investigations and analysis, with an emphasis on human disease analytics. After his first postdoctoral training at Columbia University, NY, USA, he briefly worked for a pharmaceutical company in Austria, and then worked for more than 10 years at the University of Edinburgh. This was followed by a move to the University of Glasgow, where he still holds a position, and the University of the Highlands and Islands in Inverness as principal investigator (PI) in Bioinformatics, Biostatistics and Systems Biology. Over the last three decades, he has extensively published in the area, from method development and data acquisition, databases, and big data handling, through to integrative Systems Biology approaches in order to understand complex biological systems. His investigations covered areas in neuroscience, renal complications, cardiovascular disorders, pulmonary and autoimmune diseases, as well as muscle wasting and cancer. He is the lead of the Pan-omics Database Initiative.

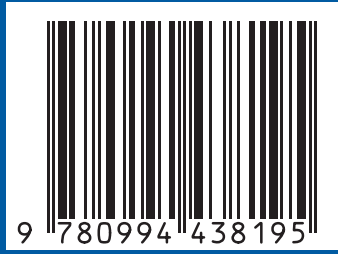

